\title{
CORRELAÇÃO ENTRE COMPONENTES DE PRODUÇÃO DE SOJA BRS TRACAJÁ E DIFERENTES DENSIDADES DE PLANTAS NO CERRADO RORAIMA
}

\author{
Oscar Jose Smiderle ${ }^{1}$, Aline das Graças Souza ${ }^{2 *}$, Vicente Gianluppi ${ }^{1}$, Daniel Gianluppi ${ }^{1}$, Kelly Naiane \\ Andrade Costa ${ }^{3}$, Hananda Hellen da Silva Gomes ${ }^{4}$
}

\begin{abstract}
RESUMO - A produtividade de uma cultura é definida pela interação entre a planta, o ambiente de produção e o manejo. Objetivou-se determinar as correlações entre os componentes da produção com diferentes densidades de plantas da soja BRS Tracajá. O experimento foi conduzido em delineamento de blocos ao acaso com quatro densidades $\left(8,10,12,14\right.$ plantas $\mathrm{m}^{-1}$ linear) e quatro repetições. As parcelas foram constituídas por quatro linhas de cinco metros de comprimento e espaçadas de 0,50 metros. Para avaliação das variáveis propostas coletaram-se as plantas da área útil (duas linhas centrais de $4 \mathrm{~m}$ lineares) por parcela. As variáveis avaliadas foram altura da planta, altura de inserção da primeira vagem, diâmetro do caule, número de nós na haste principal, número de vagens total por planta, número médio de grãos por planta, numero de vagens nos últimos 4 nós, número médio de grãos, massa seca de planta, massa seca dos grãos nos últimos 4 nós, massa seca de grãos, total da massa seca de grãos, massa seca da casca nos últimos 4 nós, massa seca da casca, total de massa seca de cascas, índice de colheita aparente, estimativa de produtividade, relação grãos nos últimos 4 nós e total (\%), massa de 100 sementes, número total de grãos. Para obter maior produtividade de grãos é indicada a população com 12 plantas $\mathrm{m}^{-1}$ de fileira. A massa de 100 sementes apresenta correlação com coeficiente de $0,8<\mathrm{r}<1,0$ com a massa seca de grão e estimativa de produtividade em diferentes densidades de plantas $\mathrm{m}-1$ linear.

Palavras chave: Glycine max, grãos, produtividade.
\end{abstract}

\section{CORRELATION BETWEEN YIELD COMPONENTS OF BRS TRACAJÁ SOYBEAN AND DIFFERENT PLANT DENSITIES ON RORAIMA CERRADO}

\begin{abstract}
A crop yield is defined by the interaction among the plant, the production environment and the management. It was aimed to determine the correlations between the yield components at different plant densities of BRS Tracajá soybean. The experiment was conducted in a randomized block design with four densities 8, 10, 12, 14 plants per linear meter with four replications. The plots consisted of four rows of five meters long and spaced 0.50 meters apart. For the evaluation of the proposed variables, plants in the useful area (two central lines of $4 \mathrm{~m}$ ) of plot were collected. The evaluated variables were plant height, first pod insertion height, stem diameter, number of nodes in the main stem, number of total pods per plant, average number of grains per plant, number of pods on the last 4 nodes, average number of grains on the last 4 nodes, dry mass of grains, total dry mass of grains, dry mass of the bark on the last 4 nodes, dry mass of the bark, total dry mass of the bark, apparent harvest index, yield estimate, grain ratio on the last 4 nodes and total (\%), mass of 100 seeds, total number of grains. To obtain greater grain, the population with 12 plants per meter is indicated. The mass of 100 seeds presents a high magnitude correlation with the dry mass of grain and yield estimate at different plant densities per meter.
\end{abstract}

Keywords: Glycine max, grains, yield.

\footnotetext{
${ }^{1}$ Pesquisador, Embrapa Roraima.

${ }^{2}$ Professora, Instituto Federal de Roraima, Amajari-RR. Contato: aline.souza@ifrr.edu.br

${ }^{3}$ Estudante de Agronomia - Faculdade Roraimense de Ensino Superior - FARES.

${ }^{4}$ Estudante de Agronomia - Universidade Federal de Roraima - UFRR.
} 


\section{INTRODUÇÃO}

O Brasil tem apresentado, nas últimas safras, desempenho crescente em termos de produtividade e produção de grãos de soja (Glycine max L.) e a base dessa pirâmide produtiva esta associada à utilização de sementes de qualidade comprovada. Na safra 2016/ 2017, a taxa de utilização de sementes de soja no Brasil foi de $71 \%$, frente a uma demanda potencial de 22.565 .170 t de sementes (Abrasem, 2018).

O manejo para obtenção de altas produtividades na cultura da soja é traduzido na interação clima, planta e solo, propondo o uso eficiente e racional dos fertilizantes (Smiderle et al., 2016 a).

O cerrado de Roraima, apresenta clima tipo Aw tropical chuvoso (Smiderle et al., 2018), com precipitação pluvial média anual de aproximados $1800 \mathrm{~mm}$ e concentração de mais de $80 \%$ das chuvas entre os meses de abril a setembro e estiagem de até 120 dias (Smiderle et al., 2017a), favorecendo o plantio de soja no estado (Silva et al., 2017).

A cultura da soja (Glycine max L.) vem sendo implantada no estado de Roraima nas áreas de cerrado e mata de transição (Nechet et al., 2008). A safra ocorre de maio a setembro, coincidindo com o período chuvoso da região (Smiderle et al., 2017 b). Em função da localização geográfica e de sua diferente época de semeadura (maio a junho) em relação ao restante do país (outubro a dezembro) ainda há pouca informação referente aos componentes de produção e arranjos espaciais de plantas de soja da cultivar tracajá.

Na busca de melhores práticas, a população adequada de plantas e o manejo do solo, são fatores determinantes para ajuste do arranjo das plantas de soja, pois influenciam nos componentes de produção especialmente número de vagens/planta e de grãos/ vagem, pois estes, se correlacionam diretamente com a produtividade de grãos, conforme Smiderle et al. (2016). Essa correlação lhe confere potencial para base de seleção indireta de genótipos mais produtivos (Procópio et al., 2013).

O número de vagens/planta e de grãos/vagens são os dois componentes mais importantes da produtividade de grãos de soja, uma vez que alterações nesses componentes são responsáveis diretos pelo ajuste da produtividade, podendo ocorrer com a modificação na população de plantas (Smiderle et al.,
2016). Entretanto, sabe-se que existe variabilidade em tais componentes entre indivíduos de uma população em função de vários fatores, que vão desde a implantação da cultura (influenciando o número de plantas por área), até a disponibilidade de nutrientes (modificada pelo arranjo de plantas), que afetam os demais componentes (número de vagens/planta, número de grãos/vagem e a massa de grãos).

A cv. BRS Tracajá apresenta importantes características tais como crescimento semi-determinado, altos porcentuais de germinação, emergência e vigor das plântulas (Smiderle et al., 2016; Lima et al., 2014), sendo uma cultivar de soja com grande potencial de uso para área de cerrado no estado de Roraima, fato que estimulou o desenvolvimento do presente estudo.

Diante desse contexto, o trabalho foi realizado com objetivo de avaliar as correlações entre os componentes da produção com diferentes densidades de plantas da cultivar BRS Tracajá no cerrado de Roraima.

\section{MATERIALE MÉTODOS}

O experimento foi instalado no Campo Experimental Água Boa, da Embrapa Roraima, situado no Município de Boa Vista, à margem esquerda da BR174, Km 25, sentido Boa Vista - Manaus/AM, tendo como coordenadas geográficas: $2^{\circ} 23^{\prime} 45,31$ " de latitude norte e $60^{\circ} 58^{\prime} 44,34 "$ de longitude oeste. O solo é classificado como Latossolo Amarelo Distrófico, de textura média, quimicamente pobre e com baixos teores de matéria orgânica conforme dados da camada de $0-15 \mathrm{~cm}$ (argila $=15 \% ; \mathrm{MO}=0,34 \%$; $\mathrm{S}=0,21 \mathrm{me} / 100 \mathrm{~g} ; \mathrm{Al}=0,40 \mathrm{me} / 100 \mathrm{~g} ; \mathrm{CTC}=1,20 \mathrm{me} /$ 100 ; $V=18 \%$; e $m=66 \%$. O fósforo natural chega a 0,11 mg/100g de $\mathrm{P}_{2} \mathrm{O}_{5}$ e o $\mathrm{K}$ a 3,5 mg/100g de solo (RADAMBRASIL, 1975).

Para instalação do ensaio foi realizada adubação com $450 \mathrm{~kg} \mathrm{ha}^{-1}$ de adubo fórmula 4-24-12, que continha: $3 \%$ de $\mathrm{N} ; 28 \%$ de $\mathrm{P}_{2} \mathrm{O}_{5}$; $9 \%$ de $\mathrm{K}_{2} \mathrm{O} ; 10 \%$ de $\mathrm{Ca} ; 8 \%$ de S; 0,3\% de Zn; 0,3\% de Mn; 0,12\% de B; e, 0,12\% de $\mathrm{Cu}$, acrescido de $120 \mathrm{~kg} \mathrm{ha}^{-1}$ de $\mathrm{K}_{2} \mathrm{O}$ (cloreto de $\mathrm{K}, 60 \%$ de $\mathrm{K}_{2} \mathrm{O}$ ) e, $50 \mathrm{~kg} \mathrm{ha}^{-1}$ de FTE BR12, aos 20 dias após a emergência das plântulas. Foram utilizadas sementes da cultivar BRS tracajá, pertencente ao grupo de materiais de crescimento semi-determinado e maturidade relativa 8.3. As sementes foram tratadas e inoculadas conforme o sistema de produção de soja para Roraima (Smiderle et al., 2009). 
A quantidade de sementes utilizadas foi de 20 sementes/m linear, suficientes para após desbaste, obter as densidades de 8, 10,12, 14 plantas $\mathrm{m}^{-1}$ linear. Dez dias após a emergência (DAE) foi realizado desbaste das plantas com corte rente ao solo. O controle de pragas, doenças e plantas invasoras seguiu as recomendações do sistema de produção. Aos 25 DAE das plantas realizou-se adubação de cobertura com $100 \mathrm{~kg} \mathrm{ha}^{-1}$ de cloreto de potássio $\left(60 \%\right.$ de $\left.\mathrm{K}_{2} \mathrm{O}\right)$.

O experimento foi conduzido em delineamento de blocos ao acaso com quatro densidades 8, 10, 12, 14 plantas $\mathrm{m}^{-1}$ linear, com quatro repetições. As parcelas foram constituídas por quatro linhas de cinco metros de comprimento e espaçadas de 0,50 metros. Para avaliação das variáveis propostas coletaram-se as plantas presentes na área útil (duas linhas centrais com 4 metros lineares) na parcela.

Nas plantas colhidas na área útil (80 por tratamento) foram determinados individualmente os parâmetros: altura de planta, altura de inserção da primeira vagem, diâmetro do caule, número de nós na haste principal, número de hastes por planta, número de vagens total por planta, número de grãos por vagem. A determinação da altura de planta e de inserção da primeira vagem foi realizada com auxílio de régua milimétrica, enquanto o diâmetro do caule foi medido com auxílio de paquímetro digital. As contagens e trilhagem das vagens foram realizadas manualmente com pesagem de plantas, cascas e grãos e determinado o número de grãos/planta além de estabelecido o índice de colheita aparente (ICA). Este foi obtido pela divisão da massa total de grãos pela massa total da parte aérea em 80 plantas analisadas.

O rendimento de grãos por área foi determinado de maneira similar ao rendimento de grãos por planta, convertido em rendimento de grãos por hectare, após correção para $13 \%$ de umidade. Foram tomadas amostras para determinação da umidade dos grãos colocados em estufa a $105^{\circ} \mathrm{C}$ por 24 horas e correção do rendimento para $13 \%$, tanto de grãos planta ${ }^{-1}$ como de grãos ha ${ }^{-1}$.

Os dados experimentais foram submetidos à análise da variância, e os efeitos de tratamento avaliados pelo teste ' $F$ '. Para as comparações de médias utilizou-se o teste de Tukey à $5 \%$ de probabilidade e o fator quantitativo analisado por regressão e adotado a equação com o maior coeficiente (Ferreira, 2011). Análise de correlação de Pearson foi realizada entre as variáveis, massa de 100 sementes (M100), altura de planta (AP), número de vagens total por planta (NVT), número de galhos por planta (NGP), número total de grãos (NTG), massa seca dos grãos (MSG), total da massa seca de grãos (TTMSG), índice de colheita aparente (ICA), estimativa de produtividade (EPROD, $\mathrm{kg} \mathrm{ha}^{-1}$ ), relação grãos nos últimos 4 nós e total (\%), massa de 100 sementes (M100), número total de grãos (NTG) em diferentes densidades por planta $\mathrm{m}^{-1}$, utilizando o Software R (2014).

\section{RESULTADOS E DISCUSSÃO}

As características número de vagens total por planta (NVT), número de vagens nos últimos 4 nós (NV4un), número médio de galhos por planta (NGP), massa seca dos grãos (MSG), total da massa seca de grãos (TTMSG), massa seca da casca (MSC), total de massa seca das cascas (TTMSC), índice de colheita aparente (ICA), número total de grãos (NTG) se mostraram significativamente maiores na semeadura realizada com 8 plantas $\mathrm{m}^{-1}$ na fileira (Tabela 1 ). Em contrapartida a densidade com 8 plantas $\mathrm{m}^{-1}$ a estimativa de produtividade por planta diminui, o que, segundo Garcia (1992), ocorre por causa da capacidade da soja em ajustar os componentes de produção.

De fato, na produção por planta com a densidade de 12 e 14 plantas $\mathrm{m}^{-1}$, ocorreram maior produtividade por área, possivelmente devido à melhor distribuição espacial das plantas, o que também contribuiu para o aumento da massa 100 sementes (Tabela 1). Somado a isso, a estimativa de produtividade correlacionouse positivamente e com alta magnitude com a massa de 100 sementes (Tabela 2). Segundo Pierozan Junior (2012), a produtividade da soja é definida pelo número de vagens por área, juntamente pelo número de sementes por vagem e pela massa de 100 sementes.

Quanto à estimativa de produtividade (Figura 1A) a análise de regressão revelou efeito quadrático com coeficiente de determinação $\mathrm{R}^{2} 0,99$, sendo assim as maiores estimativas de produtividade foram contidas no intervalo de 4.193 a $4.026 \mathrm{~kg} \mathrm{ha}^{-1}$, obtidas com as densidades 12 e 14 plantas $\mathrm{m}^{-1}$ linear, respectivamente ( 240 a 280 mil plantas ha- ${ }^{-1}$ ). Neste estudo, constatou-se que a densidade com 12 plantas $\mathrm{m}^{-1}$ apresentou a capacidade de manter a produtividade de grãos com 18,77\% superior em relação à densidade com 8 plantas $\mathrm{m}^{-1}$. Somado a isso, a altura de planta com $84,24 \mathrm{~cm}$, apresentou máxima produtividade (4.122 
Tabela 1 - Valores médios obtidos para altura de planta (AP, cm), altura de inserção da primeira vagem (AIPV, $\mathrm{cm}$ ), diâmetro do caule (DC, mm), número de nós na haste principal (NNH), número de vagens total por planta (NVT), número médio de galhos por planta (NGP), número de vagens nos últimos 4 nós (NV4un), numero médio de grãos (NG 4 un), massa seca de planta (MSP, g), massa seca dos grãos nos últimos 4 nos (MSG, 4un), massa seca dos grãos (MSG), total da massa seca de grãos (TTMSG), massa seca da casca nos últimos 4 nos (MSC 4un), massa seca da casca(MSC), total de massa seca das cascas (TTMSC), índice de colheita aparente (ICA), estimativa de produtividade (EPROD, kg ha-1), massa de 100 sementes (M100), número total de grãos (NTG), obtidos em quatro densidades de plantas de soja BRS Tracajá cultivada em área de cerrado em Boa Vista, Roraima em 2017

\begin{tabular}{|c|c|c|c|c|c|c|c|}
\hline Densidade & AP & AIPV & DC & $\mathrm{NNH}$ & NVT & NGP & NV4un \\
\hline $8 * *$ & $63,11 \mathrm{c}^{*}$ & $8,81 \mathrm{~b}$ & 7,82 a & 15,98 a & 89,43 a & 3,45 a & $17,90 \mathrm{a}$ \\
\hline 10 & 79,94 b & $10,43 \mathrm{a}$ & 7,64 a & 16,17 a & $67,88 \mathrm{~b}$ & $2,69 \mathrm{~b}$ & $15,15 b$ \\
\hline 12 & 84,34 a & 11,13 a & $7,40 \mathrm{a}$ & 16,10 a & 62,31 bc & $2,12 \mathrm{c}$ & $15,80 \mathrm{~b}$ \\
\hline 14 & 89,88 a & 11,22 a & 7,40 a & 16,34 a & 58,48 с & $1,83 \mathrm{c}$ & $15,73 \mathrm{~b}$ \\
\hline \multirow[t]{2}{*}{$\mathrm{CV} \%$} & 3,44 & 5,48 & 3,96 & 2,95 & 6,25 & 10,77 & 5,39 \\
\hline & NG4un & MSP & MSG4un & MSG & TTMSG & MSC4un & MSC \\
\hline 8 & 41,34 a & 13,014 a & 5,55 a & 23,68 a & 29,23 a & $2,18 \mathrm{a}$ & 8,64 a \\
\hline 10 & $34,05 \mathrm{ab}$ & $12,930 \mathrm{a}$ & $4,22 \mathrm{~b}$ & $17,56 \mathrm{~b}$ & $21,78 \mathrm{~b}$ & 1,99 a & 5,85 b \\
\hline 12 & $36,88 \mathrm{~b}$ & $12,232 \mathrm{a}$ & $4,94 \mathrm{ab}$ & 15,32 bc & $20,26 \mathrm{~b}$ & 1,99 a & 5,15 b \\
\hline 14 & $35,50 \mathrm{~b}$ & 13,040 a & 5,49 a & $14,01 \mathrm{c}$ & $19,83 \mathrm{~b}$ & 2,26 a & $4,67 \quad b$ \\
\hline \multirow[t]{2}{*}{ CV\% } & 7,16 & 9,18 & 9,37 & 10,34 & 10,32 & 8,75 & 11,27 \\
\hline & TTMSC & ICA & EPROD & M100 & NTG & & \\
\hline 8 & 10,82 a & 0,55 a & 3269,9 b & $13,43 \mathrm{~b}$ & 217,56 a & & \\
\hline 10 & $7,83 \mathrm{~b}$ & $0,51 \mathrm{~b}$ & $3918,0 \mathrm{ab}$ & $12,37 \mathrm{~b}$ & $175,74 \mathrm{~b}$ & & \\
\hline 12 & $6,89 \mathrm{~b}$ & $0,51 \mathrm{~b}$ & 4192,6 а & $13,41 \mathrm{~b}$ & $157,27 \mathrm{c}$ & & \\
\hline 14 & $6,75 \mathrm{~b}$ & $0,50 \mathrm{~b}$ & 4025,7 a & $15,47 \mathrm{a}$ & $127,86 \mathrm{~d}$ & & \\
\hline CV\% & 11,27 & 7,42 & 2,00 & & 5,86 & & \\
\hline
\end{tabular}

$\mathrm{kg} \mathrm{ha}^{-1}$ ) quando cultivada com 12 plantas $\mathrm{m}^{-1}$ linear (Tabela 1).

Assim, a utilização desta densidade permite maior expressão do potencial produtivo das plantas, pelo melhor aproveitamento dos fatores do meio e dos insumos aplicados. Estes resultados possibilitam economia de mais de $90 \%$ de sementes, em comparação com a densidade de 22 plantas $\mathrm{m}^{-1}$ linear, indicada na literatura por Smiderle et al. (2016) para a cultivar BRS 8581.

A relação do número de grãos nos últimos 4 nós da planta com o total de grãos (\%) apresentou ajuste linear $(\mathrm{P}<0,05)$, em função das densidades por plantas $\mathrm{m}^{-1}$ linear (Figura 1B), provavelmente devido ao número total de grãos (NTG). Tal fato pode ser atribuído devido às correlações de alta magnitude entre tais variáveis (Tabela 2). A correlação é considerada de alta magnitude quando o coeficiente de correlação varia de 0,5 $<\mathrm{r}<0,7$ (Souza et al., 2016).
De acordo com Franchini et al. (2015) os índices de colheita real e aparente (incluindo ou não as folhas senescentes, respectivamente) na cultura da soja indicam que o índice de colheita mensurado apenas com base na biomassa na maturação pode refletir a capacidade de alocação de biomassa nos grãos por planta, uma vez que no presente estudo observou- se moderada correlação entre ICA e NGP (Tabela 2), sendo considerada correlação moderada quando o coeficiente de correlação varia de 0,5 $<\mathrm{r}<0,7$ (Souza et al., 2016).

Observou-se, entretanto, leve tendência do número total de grãos aumentarem com a densidade de 12 plantas $\mathrm{m}^{-1}$. Somado a isso, o NVT se correlacionou positivamente com o NTG, apresentando correlações de alta magnitude (Tabela 2). Provavelmente, tal correlação ocorreu, em virtude do aumento da eficiência de utilização da energia solar, que com o aumento da uniformidade foi melhor distribuída sobre as folhas, resultando em maior concentração de assimilados nos grãos. 


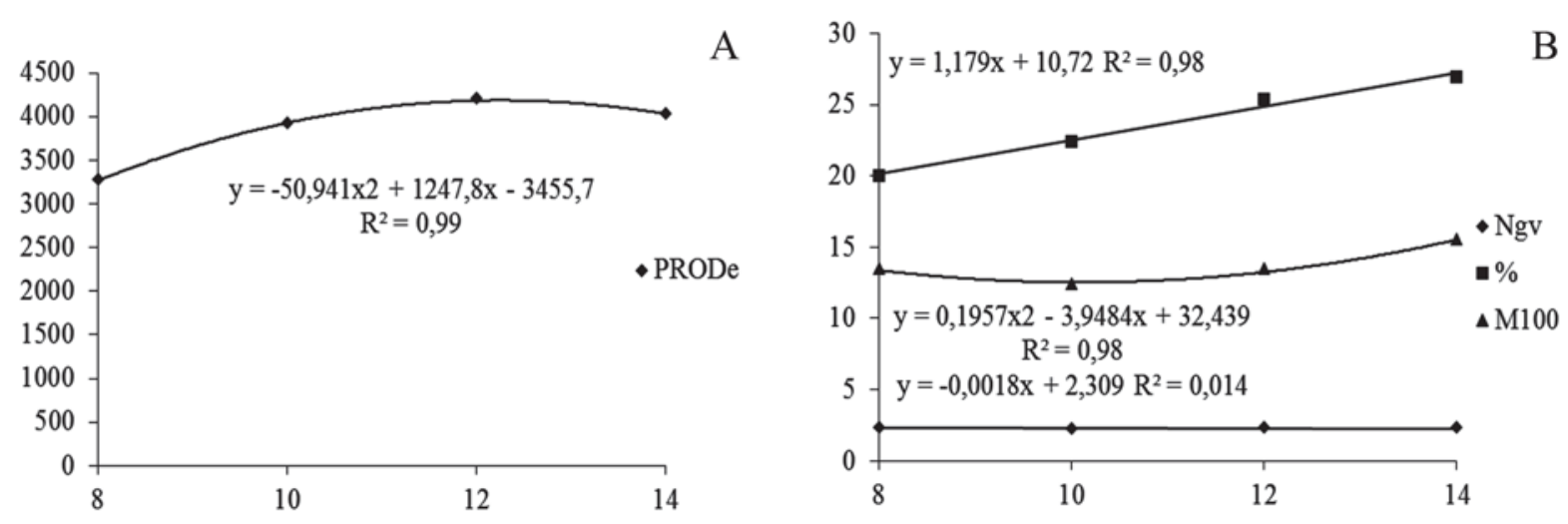

Figura 1 - Valores médios de produtividade estimada (A), número de grãos por vagem (NGV), relação entre grãos nos últimos 4 nós e total (\%) e massa de 100 sementes (B) da soja BRS Tracajá obtida em cerrado de Roraima, 2017

Tabela 2 - Matriz de coeficientes* de correlação de Pearson entre as variáveis, massa de 100 sementes (M100), altura de planta (AP), número de vagens total por planta (NVT), número de galhos por planta (NGP), número total de grãos (NTG), massa seca dos grãos (MSG), total da massa seca de grãos (TTMSG), índice de colheita aparente (ICA), germinação (\%) e estimativa de produtividade (EPROD, kg ha-1) em diferentes densidade de planta $\mathrm{m}^{-1}$ cultivada em cerrado de Roraima, safra 2017

\begin{tabular}{|c|c|c|c|c|c|c|c|c|c|c|}
\hline Variáveis & MS100 & AP & NVT & NGP & NTG & MSG & TTMSG & ICA & G\% & EPROD \\
\hline MS100 & - & $0,89^{*}$ & $0,81^{*}$ & $0,76^{*}$ & 0,95 & $0,95^{*}$ & $0,98 *$ & $0,87 *$ & $0,79 *$ & $0,91^{*}$ \\
\hline AP & - & - & $0,79^{*}$ & $0,82^{*}$ & 0,92 & $0,77^{*}$ & $0,72 *$ & $0,83 *$ & $0,69 *$ & $0,87^{*}$ \\
\hline NVT & - & - & - & 0,89 & 0,91 & $0,82^{*}$ & $0,82 *$ & $0,79 *$ & $0,72 *$ & $0,88^{*}$ \\
\hline NGP & - & - & - & - & 0,86 & $0,71^{*}$ & $0,79 *$ & $0,68 *$ & $0,69 *$ & $0,59 *$ \\
\hline NTG & & & & & - & 0,95 & 0,92 & $0,78 *$ & $0,89 *$ & $0,95^{*}$ \\
\hline MSG & - & - & - & - & & - & $0,82 *$ & $0,56 *$ & $0,62 *$ & $0,81^{*}$ \\
\hline TTMSG & - & - & - & - & & - & - & $0,62 *$ & $0,68 *$ & $0,93 *$ \\
\hline ICA & & & & & & & & - & $0,63 *$ & $0,91 *$ \\
\hline G\% & & & & & & & & & - & $0,75^{*}$ \\
\hline EPROD & & & & & & & & & & - \\
\hline
\end{tabular}

Este comportamento mostrou que a soja é uma planta que consegue se adaptar a diferentes arranjos espaciais. Porém este comportamento é variável em função das características de cada cultivar como ciclo biológico, ciclo da planta, hábito de crescimento e período juvenil, pois Tourino et al. (2002) utilizando a cultivar CAC-1, verificaram aumento linear da produtividade com a redução da densidade de plantas na linha, obtendo o máximo rendimento com 10 plantas por metro, por outro lado Cruz et al. (2016) trabalhando com a cultivar Anta 82 semi determinada obtiveram resultados similares aos do presente trabalho. Para Smiderle et al. (2016) a soja possui alta habilidade em compensar menores densidades de plantas, principalmente formando maior número de legumes por indivíduo. No entanto, essa capacidade se dá até determinado limite conforme foi observado na presente pesquisa.

\section{O N C L U S Õ E S}

Para obter maior produtividade de grãos são indicadas 12 plantas $\mathrm{m}^{-1}$ linear de fileira, equivalente a população de 240 mil plantas por hectare.

A massa de 100 sementes apresenta correlação de alta magnitude com a massa seca de grão e estimativa de produtividade em diferentes densidades de plantas $\mathrm{m}^{-1}$ linear. 


\section{AGRADECIMENTOS}

Pela concessão de bolsas de Produtividade em Pesquisa.

Os autores agradecem ao Conselho Nacional de Desenvolvimento Científico e Tecnológico (CNPq) e a Coordenação de Aperfeiçoamento de Pessoal de Nível Superior (CAPES).

\section{LITERATURACITADA}

ABRASEM. Estatísticas. Disponível em: <http:/ /www.abrasem.com.br/site/estatisticas/\# >Acesso em: 26 março .2018.

CRUZ, S.C.S.; SENA-JUNIOR, D.G.; SANTOS, D.M.A. et al. Cultivo de soja sob diferentes densidades de semeadura e arranjos espaciais. Revista de Agricultura Neotropical, v.3, n.1, p.1-6, 2016.

GARCIA, A.; PÍPOLO, A. E.; LOPES, I. O. N. Y. et al. Instalação da lavoura de soja: época, cultivares, espaçamento e população de plantas. Circular técnica, Embrapa, Londrina, PR, 2007.

FERREIRA, D.F. Sisvar: a computer statistical analysis system. Ciência e

Agrotecnologia, v.35, n.6, p.1039-1042, 2011.

FRANCHINI, C.J.C.; JUNIOR, A.A.B.; DEBIASI, H.Y. et al. Desempenho da soja em consequência de manejo de pastagem, época de dessecação e adubação nitrogenada.

Pesquisa Agropecuária Brasileira, v.50, n.12, p.1131-1138, 2015.

LIMA, J.M.E.; SMIDERLE, O.J.; ALVES, J.M.A. et al. Potencial fisiológico de sementes de soja-hortaliça cultivar BRS 258, produzidas com diferentes adubações e armazenadas. Acta Iguazu, v.6, n.1, p.96-106, 2017.

LIMA, J.M.E.; SMIDERLE, O.J.; ALVES, J.M.A. et al. Tipos de adubação e épocas de avaliação na sanidade e viabilidade de sementes de sojahortaliça. RevistaAgro@mbiente,v.8, n.2, p.244-252, 2014.
NECHET, K.L.; HALFELD-VIEIRA, B.A.; GIANLUPPI, V. et al. Reação de cultivares de soja à mela (Thanatephorus cucumeris) em campo em dois estádios de desenvolvimento das plantas.

Summa Phytopathologica, v.34, n.3, p.277279, 2008.

PROCÓPIO, S.O.; BALBINOT JUNIOR, A.A.; DEBIASI, H. et al. Plantio cruzado na cultura da soja utilizando uma cultivar de hábito de crescimento indeterminado. Revista Ciências Agrárias, v.56, n.4, p.319-325, 2013.

RADAMBRASIL. Projeto RadamBrasil: Levantamento de Recursos Naturais. Rio de Janeiro: Departamento Nacional de Produção Mineral, 1975. 260p.

SILVA, T.A.; SILVA, P.B.; SILVA, E.A.A. et al. Condicionamento fisiológico de sementes de soja, componentes de produção e produtividade.

Ciência Rural, v.46, n.2, p.227-232, 2016.

SMIDERLE, O.J.; MARINHO, J.; GONÇALVES, J. et al. Colheita e armazenamento de grãos e sementes. In: ZILLI, J.E.; VILARINHO, A.A.; ALVES, J.M.A. (Eds.) A cultura do feijão-caupi na Amazônia brasileira. Boa Vista, RR: Embrapa Roraima, p.327-356, 2009.

SMIDERLE, O.J.; SOUZA, A.G.; ALVES, J.M.A. et al. Physiological quality of cowpea seeds for different periods of storage. Revista Ciência Agronômica, v.48, n.5, p.817-823, 2017a.

SMIDERLE, O.J.; LIMA-PRIMO, H.E.; BARBOSA, H.D. et al. Effect of defoliation on production components at different growth stages of cowpeas. Revista Ciência Agronômica, v.48, n.5, p.840-847, 2017b.

SMIDERLE, O.J.; GIANLUPPI, D.; SOUZA, A.G. Variability among BRS 8381 soybean (Glycine max (L.) Merrill.) yield components under different liming rates and sowing densities on a savanna in Roraima, Brazil. Revista Colombiana de Investigaciones Agroindustriales, n.1, v.2, p.49-55, 2016.

SMIDERLE, O.J.; SOUZA, A.G.; CAMPOS, L.S. et al. Qualidade fisiológica de sementes de feijãocaupi obtidas em residual alternativos de adubações. Revista Congrega, v.2, n.1, p.217224, 2016. 
SMIDERLE, O.J.; SOUZA, A.G.; PEDROZO, C.A. et al. Correlation between mass and vigor of Pochota fendleri (Malvaceae) seeds stored in different environments. Revista de Ciências Agrárias, v.41, n.1, p.93-99, 2018.

SOUZA, A.G.; SMIDERLE, O.J.; SPINELLI, V.M. et al. Correlation of biometrical characteristics of fruit and seed with twinning and vigor of Prunus persica rootstocks. Journal of Seed

Science, v.38, n.4, p.322-328, 2016.
R Core Team. R: A language and environment for statistical computing. Áustria: R Foundation for Statistical Computing, 2014.

TOURINO, M.C.C.; REZENDE, P.M.; SALVADOR, $\mathrm{N}$. Espaçamento, densidade e uniformidade de semeadura na produtividade e características agronômicas da soja. Pesquisa

Agropecuária Brasileira, v.37, n.8, p.10711077, 2002.

Recebido para publicação em 20/11/2018 e aprovado em 12/3/2019. 